\title{
El impacto de la capacidad de absorción potencial del conocimiento sobre la innovación en marketing
}

\section{The impact of the potential absorption capacity of knowledge on innovation in marketing}

\author{
Diana Leidy Guerrero-Sánchez * \\ Facultad de Ciencias Empresariales, Fundación Universitaria Lumen GentiumUnicatólica, Colombia
}

Recibido el 12 de noviembre de 2018; aceptado el 27 de enero de 2020

Disponible en Internet el: 6 de marzo de 2020

\section{Resumen}

Los recursos comerciales y de marketing son estrategias disponibles que promueven el proceso de innovación, si también se vinculan a las fuentes de conocimiento externo. El propósito de este artículo es analizar el efecto positivo o negativo de la capacidad de absorción potencial del conocimiento externo (CAP) y de cada una de sus dimensiones (Adquisición y asimilación) sobre la innovación en marketing. La muestra utilizada está conformada por 134 empresas del sector turístico (hoteles, restaurantes y agencias de viajes) de Colombia, y para contrastar las hipótesis del estudio, se utilizó un modelo estructural, mediante el programa Smart -PLS. Los hallazgos de este estudio muestran que la capacidad absorber potencialmente nuevo conocimiento en las empresas turísticas, influye positivamente sobre los resultados de la innovación en marketing debido a la importancia que ejerce la nueva información en la adopción de nuevas estrategias, de promoción, comercialización y venta de productos y/o servicios turísticos, concluyendo como tal, que la capacidad de absorción potencial del conocimiento externo una vez se adquiere y asimila por los individuos emerge de manera significativa para promover resultados de innovación en marketing en las estrategias organizativas de las empresas turisticas.

Código JEL: Z31, Z33, Z39

Palabras clave: Capacidad de absorción; Conocimiento externo; Innovación en marketing; Industria turística

\footnotetext{
*Autor para correspondencia.

Correo electrónico: dlguerrero@unicatolica.edu.co (D.L. Guerrero-Sánchez).

La revisión por pares es responsabilidad de la Universidad Nacional Autónoma de México. 


\begin{abstract}
Commercial and marketing resources are available strategies that promote the innovation process if they are also linked to external knowledge sources. The purpose of this article is to analyze the positive or negative effect of the potential absorption capacity of external knowledge (CAP) and each of its dimensions (Acquisition and assimilation) on marketing innovation. The sample used is made up of 134 companies of Colombia, tourism sector (hotels, restaurants and travel agencies) and to test the hypotheses of the study, a structural model was used, using the Smart-PLS program. The results of this study show that the ability to potentially absorb new knowledge in tourism businesses positively influences the results of marketing innovation due to the importance of new information in the adoption of new strategies, promotion, marketing, and sales. of tourism products and / or services, concluding as such, that the potential absorption capacity of external knowledge once acquired and assimilated by individuals emerges significantly to promote results of marketing innovation in the organizational strategies of tourism companies.
\end{abstract}

JEL Code: Z31, Z33, Z39

Keywords: Absorptive capacity; External knowledge; Innovation in marketing; Tourism

\title{
Introduction
}

La capacidad de absorción es un determinante dinámico importante para desarrollar la capacidad de innovación de una empresa (Cepeda-Carrión, Cegarra-Navarro, y Jimenez-Jimenez, 2012). A si, la necesidad de percibir los cambios del mercado y generar innovaciones como resultado de la capacidad de absorción del nuevo conocimiento externo, resulta en una ventaja competitiva si se orienta hacia los procesos de mejora continua que implican innovaciones y diferenciación (Engelman, Fracasso, Schmidt y Zen, 2017). En tal sentido, los planteamientos desde las capacidades dinámicas incita a que las empresas reconfiguren el conocimiento organizativo existente y la capacidad de absorción como antecedente de este orienta la aplicación del conocimiento externo para habilitar a las empresas a valorar, transformar y aplicar dicho conocimiento (Cohen y Levinthal, 1990).

Es así como, algunos estudios exponen cómo los procesos de la adquisición y utilización del conocimiento influyen en la gestión del conocimiento y en el rendimiento competitivo de las empresas (Tomas y Wood, 2015), y la importancia que ejercen las capacidades de adquirir y asimilar el nuevo conocimiento externo, en la habilidad de absorber y potencializar la información del entorno y utilizarla para fines innovadores (Zhara y George, 2002; Forés y Camisón, 2016) asegurando además, resultados en sus estrategias de marketing (Xiong \& 
Bharadwaj, 2011). En este sentido, es posible encontrar algunos estudios sobre los resultados de la innovación a causa de la capacidad de absorber el conocimiento (Nieto y Quevedo, 2005; Arbussà y Coenders, 2007; Grimpe y Sofka, 2009; Murovec y Prodan, 2009; Rothaermel y Alexandre, 2009; Camisón y Forés 2010; Lewin, Massini y Peeters, (2011); Kostopoulos, Papalexandris, Papachroni, y Ioannou, Milwood y Zach, 2016; Zobel, 2017; Engelman, et al. 2017), canalizando aquellos que se despliegan desde las estrategias de las capacidades de innvación en marketing (Tzokas, Kim, Akbar y Al-Dajani, 2015; Najafi-Tavani, Sharifi, y Najafi-Tavani, 2016; Medase y Barasa, 2019).

Tzokas et al. (2015) plantear la interacción entre la capacidad de absorción de una empresa (ACAP) y su capacidad tecnológica y de relación con el cliente contribuyen al rendimiento organizacional, estos autores utilizan el modelo de ecuaciones estructurales para evaluar los efectos de la absorción del conocimiento externo sobre el rendimiento mediado por diferentes capacidades de marketing, y resaltan la importancia de este tipo de capacidades para desarrollar habilidades innovadoras de relación comercial. Por otro lado, Medase y Barasa, (2019) investigan cómo la capacidad de absorción y las capacidades de comercialización están asociadas con la comercialización de productos innovadores en empresas de fabricación y servicios.

Por su parte, Najafi-Tavani et al. (2016) indica que las capacidades de marketing son impulsadas por dos formas en el contexto de la innovación, por una parte, la comercialización del nuevo producto que implica las investigaciones de mercado y publicidad, y por otro lado, aquellas actividades de innovación en marketing que implican la modificación y adaptación del producto al mercado, tales como: cambios en imagen, empaque, diseño, nuevas líneas de distribución entre, otras.

Así pues, se encuentran algunas investigaciones que analizan el efecto de la capacidad de absorción del conocimiento externo sobre algunos tipos de innovación, específicamente en procesos (Murovec y Prodan, 2009), tecnológica (Petraite, 2010; Cepeda-Carrion, et al. 2012), y en productos y/o servicios (Stock, et al. 2001; Hervas-Oliver y Albors-Garrigos, 2009; Hervas-Oliver, Garrigos, y Gil-Pechuan, 2011; Kostopoulos, et al. 2011; Aliasghar, Rose y Chetty, 2019), siendo aún más frecuentes en términos de aplicación las investigaciones que se abordan desde el sector industrial (Aguilar-Olaves, Herrera, Clemenza, 2012), relegándose así, los estudios en otros sectores económicos como el turismo (Cruz, Guerrero y Miquel, 2018), que necesariamente requeren esfuerzos en marketing para la comercialización de productos y la prestación de los servicios turisticos. En este sentido, el objetivo de esta investigación es analizar el efecto o no de la capacidad de absorción potencial del conocimiento externo sobre la innovación en marketing en el sector turístico. Por lo anterior, la pregunta de estudio a plantear es. ¿En qué medida la Capacidad de Absorción Potencial del conocimiento externo tiene un efecto o no sobre la innovación en marketing?; y a su vez, sobre la particularidad de cada una de sus dimensiones adquisición y asimilación. 
De modo que, las investigaciones sobre la capacidad de absorción potencial del conocimiento como resultado de las innovaciones con impacto comercial/marketing, aún son limitadas desde dichas áreas de conocimiento y aún más entorno al turístico. De ahí que, las emisiones de juicios sobre dichas relaciones requieren mayor atención, ya que no existe claridad, de cómo éstas empresas pueden crear ventajas competitivas en la innovación de productos y servicios recurriendo a fuentes externas de conocimiento (Zobel, 2017; Vázquez, Fernández y Félix, 2017) y a la mejora de las capacidades de marketing (Murray y Kotabe, 2011). Señalando también, la poca atención que ha recibido la innovación en marketing y la falta de estudios empíricos que investigan, el efecto de las capacidades de absorción del conocimiento sobre este tipo de innovación no tecnológica (Guerrero-Sánchez, 2017; Zobel, 2017; Vázquez, et al. 2017; Cruz, Guerrero y Miquel, 2018).

Este artículo responde a los llamados de la literatura y contribuye en este campo, debido a que se presenta evidencia empírica del valor sobre dicha relación, en particular en el sector turístico colombiano, al analizar si la capacidad de absorción potencial del conocimiento externo tiene un impacto significativo o no sobre la innovación en marketing, variables que han sido poco explorada en el mencionado sector, aplicando modelos de Ecuaciones estructurales. Para cumplir con el propósito de este estudio, el desarrollo del artículo implica en primer lugar, los antecedentes empíricos de las variables objeto de estudio, para poder así fundamentar las hipótesis con base en la literatura previa; en segundo lugar, se presenta la metodología empleada para comprobar las hipótesis del modelo estructural; posteriormente, se muestran los resultados obtenidos. Y finalmente, se discuten los hallazgos del estudio y se plantean las principales conclusiones de la investigación.

\section{Revisión de la literatura y planteamiento de hipótesis}

\section{La capacidad de absorción del conocimiento externo y sus dimensiones (La adquisición y asimilación)}

La literatura no sólo sostiene que la capacidad de absorción del conocimiento externo es la habilidad de reconocer, identificar y adquirir el valor del nuevo conocimiento para interiorizarlo en sus procesos organizativos y explotarlo con fines de comerciales (Zhara y George, 2002; Abussa y Coenders, 2007; Ali, Kan, y Sarstedt, 2016), sino que, además, propone un marco de análisis de sus componentes, siendo Zahra y George (2002) los que robustecieron dicho concepto mediante el análisis de cada uno de sus componentes: la Capacidad de Absorción Potencial (CAP) y la Capacidad de Absorción Realizada (CAR); y cuya finalidad de la primera, es reconocer, adquirir y asimilar las necesidades reales del mercado para alcanzar un 
mejor desempeño innovador en las empresas (Eisenhardt y Martin, 2000; Morgan, Vorhiesb y Mason, 2009; Forés y Camisón, 2016; Zobel, 2017).

Para Lewin, Massini y Peeters, (2011) la capacidad de absorción potencial son habilidades que se relacionan con el entorno en busca de un nuevo conocimiento, una vez se valora la información que es adquirida y asimilada. En línea con los planteamientos de Zhara y George (2002) la capacidad de adquisición se define como el reconocer y entender el conocimiento externo potencialmente valioso que reside fuera de la organización, resaltando ambos estudios la importancia de las rutinas organizativas; y desde otra perspectiva también se manifiesta la relevancia del conocimiento previo de los individuos (cohen y Levinthal, 1990), ya que la relación de la empresas con los stakeholders permite obtener nuevos conocimientos y mejores alcances tecnológicos que sus rivales o competidores que se encuentran menos relacionados (Kostopoulos et al., 2011).

Por otro lado, Zhara y George (2002) indica que la capacidad de asimilar el conocimiento permite dirigir procesos y rutinas sobre el nuevo conocimiento adquirido ya sea analizado, procesado, interpretado, comprendido e interiorizado, puesto que la asimilación es definida como aquella capacidad que permite almacenar y gestionar eficientemente el conocimiento (Marzo de 1991) y se valora a través de la sistematización, el procesamiento, la operatividad de procesos, la intuición en la toma de decisiones, conformando como tal, estas dos dimensiones la capacidad de absorción potencial del conocimiento externo.

\section{La capacidad de absorción potencial del conocimiento externo}

Las interacciones externas a las empresas permiten captar conocimiento disponible en el entorno organizacional e integrarlo, mediante diversos mecanismos de asimilación a la dinámica de conocimiento e innovación de la propia empresa. Dichos procesos mentales de asimilación del conocimiento permiten la resolución de problemas y diferentes competencias de aprendizaje que se encuentran tanto interna como externamente a la organización Amara, Landry, y Traoré, 2008; Cohen y Levinthal, 1990; Zahra y George, 2002). Por lo tanto, las capacidades de adquirir y asimilar el nuevo conocimiento externo, habilitan la capacidad de absorber y potencializar el conocimiento del entorno (Zhara y George, 2002; Forés y Camisón, 2016). En tal sentido se plantea la siguiente hipótesis H1: La adquisición de conocimiento externo (CA1) tiene un efecto positivo sobre la asimilación del conocimiento externo (CA2). 


\section{Relación entre la capacidad de absorción potencial y la innovación en marketing}

Para Astro Spila, Rocca, Ibarra (2009) la innovación se entiende como un proceso colectivo e interactivo orientado que genera externalidades de aprendizaje, cuyas diversas formas y fuentes interactúan entre sí mediante una efectiva gestión del conocimiento, que se condensan en la resolución de problemas y la mejora de la innovación, entendiendo sus diferentes tipos: procesos, producto, tecnológico, organizacional y marketing, y donde esta última, es relevante para entender la capacidad de comercialización y posicionamiento de las organizaciones.

De modo que, la relación entre la capacidad de absorción y la capacidad de innovación de la empresa ha sido estudiada por Cepeda- Carrion, et al. (2012), al interesarse por la interacción de las empresas con el entorno, como fuente de información útil de nuevo conocimiento que genera ventajas competitivas y en las que incursionar en el mercado genera innovación, cuyas bondades permiten "la introducción de un nuevo o significativamente mejorado producto o servicio, proceso, método de organización, o de comercialización y marketing, en las prácticas internas de la empresa o en sus relaciones exteriores" (Manual de Oslo, 2005, p. 56).

Lynskey (2004) señala que para mejorar la actividad innovadora de una empresa, no sólo es importante aplicar internamente un conjunto de capacidades tratando de alcanzar este objetivo, sino también, permanecer alerta para asociarlas con fuentes externas de conocimiento. De manera que, la habilidad de una empresa para utilizar el conocimiento obtenido del exterior, se percibe a través de dos procesos secuenciales para determinar la capacidad potencial del conocimiento: primero; la adquisición del conocimiento, al reconocer y comprender el nuevo conocimiento externo a la empresa potencialmente valioso, y segundo; la asimilación, al cortejar el nuevo conocimiento y discernir el realmente valioso, con la finalidad de obtener resultados de innovación (Cohen y Levinthal, 1990; Lane, Koka y Pathak, 2006).

Entre tanto, los factores no tecnológicos de la innovación que son específicamente aplicables al turismo incluyen como parte de esta, la innovación, la comercialización y el marketing que se explican por una serie de características de la empresa como son: la dependencia del operador turístico, la localización geográfica y/o la cooperación (Tejada y Moreno, 2013) y en los que esta última característica de relacionamiento con el entorno, permite establecer los canales colaboración necesarios para adquirir y asimilar el conocimiento valioso del entorno, siendo la capacidad de absorción potencial una habilidad necesaria para reconocer esas nuevas propuestas de valor en el mercado; y en donde los efectos de las redes de innovación colaborativa en la capacidad de innovación son significativos solo en presencia de capacidad de absorción (Najafi-Tavani, et al., 2018) 


\section{La adquisición del conocimiento y la innovación en marketing}

Tidd y Bessant (2018) señalan que la capacidad de gestionar innovaciones se puede mejorar a través de la adquisición de nuevos conocimientos (tecnológicos, regulatorios y marketing) que se agregaran a la base de conocimiento de la empresa para su uso en productos y/o procesos nuevos o mejorados. En este sentido, Matti Tuominen y Saara Hyvönen (2004) plantean que las innovaciones gerenciales y tecnológicas desempeñan un papel fundamental en la comprensión de cómo se logra la superioridad competitiva en los canales de marketing en constante evolución, pues al articular estas, con el nuevo conocimiento generan relaciones o vínculos externos que incrementan la probabilidad de obtener información que al utilizarse útilmente con el conocimiento interno producen innovaciones en las empresas (Leiponen, y CE Helfat, 2010).

Por lo tanto, el nivel de colaboración con diferentes stakeholders puede mejorar las capacidades de innovación de las empresas solo si los gerentes de las mismas han desarrollado la capacidad de escanear y adquirir conocimiento externo (Najafi-Tavani, et al.2018). De tal forma que, Berkhout y Col. (2010) determinan el desarrollo y la difusión de nuevos productos innovadores provienen generalmente, de ideas relacionadas con la comprensión de los clientes potenciales y sus necesidades de marketing. Por lo anterior se expone la siguiente hipótesis. H2: La adquisición de conocimiento externo (CA1) tiene un efecto sobre la innovación en marketing (INN).

\section{La asimilación del conocimiento y la innovación en marketing}

La asimilación del conocimiento, implica desarrollar procesos mentales que se asocian a generar competencias para la resolución de problemas que permiten encontrar creativamente la solución de nuevos imprevistos que se están disponibles tanto interna como externamente en las empresas (Amara et. al, 2008; Zahra y George, 2002) impulsando así, los esfuerzos en innovar, al proveer las capacidades existentes del nuevo conocimiento y de las capacidades internas que mantienen a la organización alerta a los adelantos tecnológicos y a la asimilación de nueva tecnología desarrollada fuera de esta (Aguilar-Olaves, Herrera, Clemenza, 2012).

Por lo tanto, la introducción de nuevos productos o servicios en caminados a las estrategias de marketing, proporcionados por la capacidad de asimilar contribuye al éxito de la empresa, ya que, la vigilancia del entorno en ambientes dinámicos permite obtener una posición más competitiva para actuar sobre las necesidades reales del mercado y, por lo tanto, alcanzar un mejor desempeño que los rivales (Eisenhardt \& Martin, 2000; Morgan et al., 2009), dichos cambios generan modificaciones que implican la prestación del producto o servicio, así como las nuevas formas de promoción, comunicación y comercialización en 
el mercado. Para Cohendet y Meyer-Krahmer (2001) y Astro Spila, Rocca y Ibarra (2009) la importancia de codificar el conocimiento para la innovación permite la transformación de este en un bien económico y transferible al mercado. Sobre lo anterior, se expone como proposición que: H3: La asimilación del conocimiento externo (CA2) tiene un efecto sobre la innovación en marketing (INN).

\section{La capacidad de absorción potencial y la innovación en marketing}

Las empresas tienen la habilidad de adquirir y asimilar la nueva información externa a través de la capacidad de absorción potencial, como resultado del nuevo conocimiento externo (Cohen y Levinthal, 1990; Zhara y George, 2002;) siendo significativo también en el contexto del turismo (Williams y Shaw, 2011; King, Breen y Whitelaw, 2014), ya que los cambios se avivan desde actividades especializadas del marketing como son: la comunicación, ventas, desarrollo de producto, fijación de precios, distribución, estrategias de diferenciación en el mercado, además de los análisis y estudios sobre planes de marketing (Vorhies, Morgan y Autry, 2009).

Dicho lo anterior, la capacidad de absorción potencial mediante la adquisición y asimilación del conocimiento externo plantean constructos fiables y adecuadas medidas (Murovec y Podan, 2009) para fortalecer y mantener las relaciones hacia el exterior, con los proveedores, consumidores y demás grupos de interés (Todorova y Durisin, 2007), ello fomenta el conocimiento recientemente adquirido y asimilado, al mejorar las competencias existentes, una vez se integra nueva información en las operaciones como resultado del relacionamiento con el mercado (Zahra y George, 2002; Aguilar-Olaves, Herrera y Clemenza, 2012), que son determinantes para la innovación en marketing.

De tal forma que, el desempeño organizacional de cara a las áreas funcionales de I + D, fabricación y comercialización, contribuyen a la absorción del conocimiento potencial proveniente de diferentes fuentes de conocimiento externas (Volberda, Foss, y Lyles, 2010). Desde este punto de vista, la capacidad de absorción potencial manifiesta un proceso de aprendizaje por interacción orientado hacia la innovación a servicio de la explotación comercial (Cohen y Levinthal, 1990; Zahra y George, 2002), siendo un determinante dinámico importante para desarrollar la capacidad de innovación de las empresas (Cepeda-Carrion, Cegarra-Navarro, y Jimenez-Jimenez, 2012).

Por lo tanto, las empresas deben analizar e interpretar constantemente las demandas cambiantes del mercado y comprender rápidamente las nuevas oportunidades para traducirlas en productos y servicios competitivos orientados hacia la innovación en marketing, fundamentado que Corral de Zubielqui y col, (2016) demuestran que la ACAP tiene una influencia directa y 
positiva en los resultados de innovación, que se relacionan con las capacidades de marketing. Esta afirmación da origen a la siguiente hipótesis. H4: La capacidad de absorción potencial del conocimiento externo (CAP) tiene un efecto sobre la innovación en marketing (INN).

En razón de todo lo anterior, el modelo de investigación (figura 1) representa la relación de las hipótesis del presente estudio.

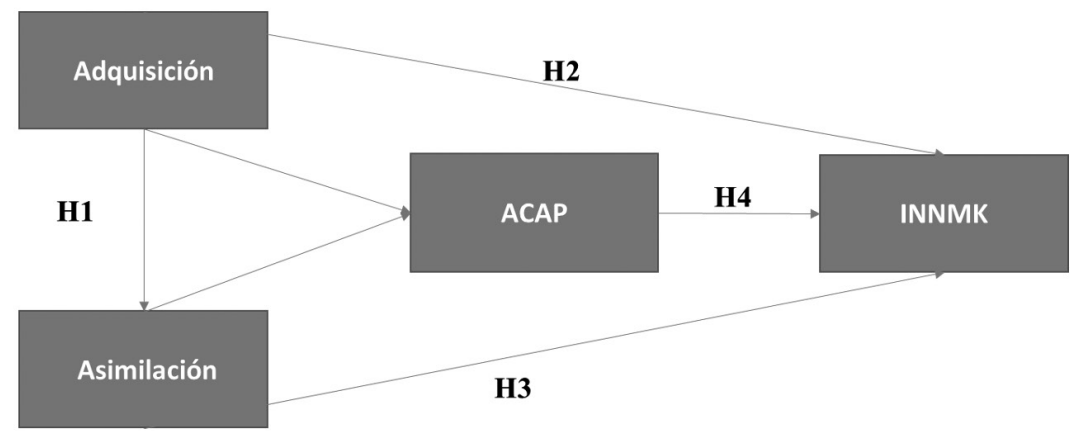

Figura 1. Modelo de la investigación que representa el planteamiento de las hipótesis del estudio. Fuente: Elaboración propia

\section{Método}

La metodología utilizada en este artículo da cuenta de la medición del impacto de la capacidad de absorción potencial del conocimiento externo sobre la innovación en marketing, tras analizar la adquisición y la asimilación como dimensiones que impactan de manera conjunta e independiente los resultados de innovación en marketing, en las empresas turísticas.

En cuanto a la recogida de información tras un proceso de muestreo estratificado, se aplicó el cuestionario a una población de 1000 empresas, dirigido a gerentes y directores de marketing de empresas turísticas de donde se obtuvieron 134 cuestionarios válidos, lo que significó una representación de los hoteles y restaurantes, en un $40 \%$ y $29 \%$ respectivamente y otras actividades del sector un $31 \%$. En el cuestionario se plantearon preguntas de opción múltiple para definir el perfil de las empresas (1-5) con relación a criterios como el tamaño y la antigüedad de las empresas encuestadas y para el caso de las variables se empleó las escalas tipo Likert de 5 puntos. Finalmente, el error muestral tomando a una población finita, fue del $8 \%$; a un nivel de confianza del $95 \%(\mathrm{Z}=1,96)$ y para $\mathrm{p}=\mathrm{q}=0,5$. 
Con respeto a los ítems de las encuestas, estos fueron adoptados de Flatten, Engelen, Zahra y Brettel, (2011) quienes cuentan con una escala aceptada en la literatura de la Capacidad de absorción del conocimiento, y que para el caso de la capacidad de absorción potencial se midieron según las dimensiones adquisición y asimilación (Tabla 1, V6-V12). Por otro lado, mediante la clasificación de los tipos de innovación del Manual de Oslo se tomó la innovación en Marketing o en comercialización aplicando la escala de medición de Camisón (2005), tal como se muestra en la Tabla 1, (V13-V19). En los dos casos, las escalas de los ítems, fueron adaptados al contexto colombiano según los resultados de la prueba piloto aplicada.

Para la evaluación del modelo de medida se utilizó como el modelo de ecuaciones estructurales, mediante la técnica Smart PLS, que permitió el estudio de las relaciones recíprocas entre variables estudiadas, incorporando el error de medida (Batista-Foguet y Coenders-Gallard, 2000), esta técnica se eligió teniendo en cuenta la pertinencia de la misma, al aceptar tamaños muéstrales pequeños, hasta mínimos de 100 (Hoyle, 1995) que no requieren una distribución normal al ser una técnica estadística no paramétrica (Hair et al., 2017; Felipe, Roldan y Leal., 2017) Considerando, además que esta técnica se basa esencialmente en el análisis causal de los modelos de investigación predictivos pocos desarrollados (Wold, 1979; Barclay et al., 1995).

Para los hallazgos del estudio, el proceso de aplicación de la técnica PLS se desarrolló en dos fases: en primer lugar, la evaluación de las escalas de medida y en segundo lugar, la evaluación del modelo estructural. En tal sentido, se desarrolló el análisis de la fiabilidad individual del ítem, la consistencia interna o fiabilidad de las escalas, el análisis de la varianza extraída media y la validez discriminante. Así, entre los planteamientos, se aplicó los sugeridos por Carmines y Zeller (1979) según el cual, un indicador deberá tener una carga igual o superior a ( $\lambda>=0.707)$ ello implica, que la varianza compartida entre el constructo y su indicador sea mayor que la varianza del error, aunque también, se consideran los parámetros de Chin (1998) al aceptar un valor $(\lambda>=0.6)$ en el tratamiento inicial de valoración en las escalas.

Finalmente, para examinar la magnitud y la significación de las relaciones entre las distintas variables planteadas en la evaluación del modelo estructural de hipótesis, se analizaron los valores (R2) y los coeficientes path ( $\beta$ ) mediante la prueba técnica no paramétrica bootstrap (Falk y Miller (1992) así como también, los constructos dependientes en el test de Stone-Geisser (Q2), según las indicaciones de Hair, Ringle y Sarstedt, (2011) y Sarstedt, Ringle y Hair, (2017). 


\section{Resultados}

Evaluación del modelo de medida

Para el análisis del modelo de medida en el proceso de depuración de los resultados del análisis de la fiabilidad individual del ítem, se mantuvieron aquellas cargas superiores a 0.7 ; y se eliminaron por el contrario los ítems inferiores a 0,6 , que, para todos los casos valorados, se presentó un solo ítem perteneciente a la escala que mide el grado de innovación en marketing (V34). Los resultados revelaron que son significativas todas las cargas de los ítems sobre su factor pronosticado $(\mathrm{p}<0,01)$ y que sus cargas estandarizadas son mayores de 0,6 (Bagozzi y Yi, 1988), siendo el promedio de las mismas superior a 0,7 (Hair, Black, Babin, Anderson y Tatham, 2005). Por lo anterior, se puedo afirmar que los ítems analizados evidencian la significancia en la fiabilidad individual de las diferentes escalas evaluadas: Adquisición, asimilación e Innovación en Marketing (Tabla 2 y 3).

\section{Fiabilidad de la escala o consistencia interna y validez convergente}

Para la evaluación de la fiabilidad de la escala de medida, los indicadores utilizados fueron el coeficiente alpha de Cronbach (Cronbach, 1951) y el índice de Fiabilidad Compuesta (Werts, Linn y Jöreskog, 1974). Tras el análisis, para todas las escalas de medición tanto los coeficientes alpha de Cronbach como los índices de Fiabilidad Compuesta, superan el valor crítico aceptado de 0,8 , tal como sugiere (Nunnally, 1978) quien recomienda para investigaciones básicas un valor estricto del 0,8 (Tabla 2), con lo cual, se puede afirmar que dichas escalas cumplen la propiedad de consistencia interna de las mismas.

Por otro lado, en este mismo análisis se evaluó la validez convergente de las escalas, que proporciona la cantidad de varianza que un constructo obtenido de sus indicadores, con relación a la cantidad de varianza debida al error de medida, calculada a través de la varianza extraída media (AVE). Según Fornell y Larcker (1981) el valor AVE debe ser superior a 0,5, lo que garantiza que más del $50 \%$ de la varianza del constructo se debe a los indicadores que lo forman, cumpliéndose para tal fin los constructos medidos, cuyos valores AVE fueron superiores a 0.6 (Tabla 2). Por lo tanto, el conjunto de indicadores evaluados representó un único constructo subyacente, que se demostraron por medio de su unidimensionalidad (Henseler, Ringle y Sinkovics, 2009). 


\section{Tabla 1}

Indicadores y cargas estandarizadas de las escalas de capacidad de adquisición, asimilación del conocimiento externo e Innovación

\begin{tabular}{|c|c|c|}
\hline \multicolumn{3}{|l|}{$(\mathrm{CA} 1+\mathrm{CA} 2+\mathrm{INN})$} \\
\hline Indicador & Carga & Valor $\mathrm{t}$ \\
\hline \multicolumn{3}{|l|}{ Capacidad de adquisición } \\
\hline $\begin{array}{l}\text { V6. La búsqueda de información relevante sobre la industria es una actividad diaria } \\
\text { en la empresa }\end{array}$ & 0,747 & 8,516 \\
\hline $\begin{array}{l}\text { V7. La dirección de la empresa motiva a los empleados a utilizar fuentes de informa- } \\
\text { ción dentro de la industria }\end{array}$ & 0,886 & 36,183 \\
\hline \multicolumn{3}{|l|}{ V8. La dirección de la empresa espera que los empleados manejen información más } \\
\hline \multicolumn{3}{|l|}{ Capacidad de asimilación } \\
\hline V9. En la empresa las ideas son comunicadas entre los departamentos. & 0,716 & 7,325 \\
\hline V10. La dirección destaca por el apoyo entre departamentos para resolver problemas. & 0,872 & 28,333 \\
\hline \multicolumn{3}{|l|}{$\begin{array}{l}\text { V11. En la empresa existe un flujo de información rápido, por ejemplo, si una unidad } \\
\text { de negocio obtiene información importante esta se comunica a todas las demás }\end{array}$} \\
\hline $\begin{array}{l}\text { V12. La dirección exige reuniones periódicas interdepartamentales para intercambiar no } \\
\text { vedades, problemas y logros. }\end{array}$ & 0,862 & 26,800 \\
\hline \multicolumn{3}{|l|}{ Innovación en marketing } \\
\hline V13. Modificación significativa de los métodos de comercialización actuales & 0,779 & 17,152 \\
\hline V14. Reducción del periodo de respuesta a un cliente o proveedor & & \\
\hline V15. Cambios significativos en el diseño del servicio & 0,824 & 21,707 \\
\hline V16. Cambios significativos en la tarificación de los productos/servicios & 0,743 & 9,666 \\
\hline V17. Introducción de nuevos canales y formatos para promocionar productos/servicios & 0,788 & 18,289 \\
\hline $\begin{array}{l}\text { V18.Desarrollo y lanzamiento de nuevas marcas, con el fin de renovar imagen o posicio } \\
\text { narse en nuevos mercados }\end{array}$ & 0,811 & 23,585 \\
\hline V19. Renovación de las marcas existentes & 0,785 & 19,679 \\
\hline
\end{tabular}

Fuente: Elaboración propia

Tabla 2

Fiabilidad y validez convergente

\begin{tabular}{lccc}
\hline \multicolumn{1}{c}{ Factor } & $\alpha$ de Cronbach & IFC & AVE \\
\hline Capacidad de adquisición (CA1) & 0,712 & 0,838 & 0,634 \\
Capacidad de asimilación (CA2) & 0,799 & 0,866 & 0,620 \\
Innovación en marketing (Inn) & 0,879 & 0,908 & 0,622 \\
\hline
\end{tabular}


Finalmente, se comprobó la validez discriminante en el modelo de medida, para el cual Fornell y Larcker (1981) indican que la varianza compartida por pares de constructos es menor que su correspondiente AVE (Tabla 3), existiendo para este caso valores (AVE) mayores a las correlaciones que presentan el constructo con el resto de constructos, siendo son positivos los resultados del modelo de medida, al demostrar que las medidas de los constructos son fiables y válidos.

Tabla 3

Validez discriminante

\begin{tabular}{lccc}
\hline & $\begin{array}{c}\text { Capacidad de } \\
\text { adquisición }\end{array}$ & $\begin{array}{c}\text { Capacidad de } \\
\text { asimilación }\end{array}$ & $\begin{array}{c}\text { Innovación en } \\
\text { marketing }\end{array}$ \\
\hline Capacidad de adquisición & 0,797 & & \\
Capacidad de asimilación & 0,600 & 0,787 & 0,789 \\
Innovación en marketing & 0,395 & 0,489 & \\
Debajo diagonal: correlaciones entre los constructos & & \\
Diagonal: raíz cuadrada de la varianza extraída media (AVE) & & \\
\hline
\end{tabular}

\section{Evaluación del modelo estructural}

Para la evaluación del modelo estructural, se analizaron las correspondientes relaciones de las hipótesis planteadas, con base a la intensidad y significatividad de los coeficientes path $(\beta)$, la varianza explicada $\left(\mathrm{R}^{2}\right)$ de las variables dependientes y su relevancia predictiva $\left(\mathrm{Q}^{2}\right)$. En tal sentido, la varianza explicada $\mathrm{R}^{2}$ expresa sí la cantidad de la varianza de las variables endógenas es explicada por los constructos que las predicen, siendo necesario que los valores $\mathrm{R}^{2}$ sean superiores a 0,10 , al considerar los resultados $0,67,0,33$ y 0,19 como sustancial, moderado y débil en su orden (Chin, 1998). Para este caso, los valores obtenidos $R^{2}=0.365$ y 0.260 (Tabla 4) arrojaron una moderada proporción en el modelo, al ser mayor a 0,10 en su valor $\mathrm{R}^{2}$.

Por otra parte, los coeficientes path estandarizados $\boldsymbol{\beta}$, miden las variables predictoras que contribuyen a la varianza explicada de las variables endógenas (Falk y Miller, 1992), para esta medición se obtuvieron, valores estadísticos $\mathrm{t} \geq 1.96$ y $\mathrm{P}$ Valores $\leq 0.05$ ), tal como se muestra en la tabla 8 , indicando los resultados la existencia en la fuerza relativa de las relaciones estadísticas entre las variables. Por otro lado, para medir la relevancia predictiva de los constructos dependientes se analizó el test de Stone-Geisser $\left(\mathrm{Q}^{2}\right)$, el cual representa que los valores observados son reproducidos por el modelo y sus parámetros estimados, en- 
tendiendo que un valor $\mathrm{Q}^{2}$ mayor que cero implica valores superiores a cero, siendo adecuada la calidad predictiva del modelo, al indicar que estas estimaciones son estables (Tabla 4).

Tabla 4

Resultados del modelo estructural: $\mathrm{R}^{2} \rightarrow \mathrm{Q}^{2}$

\begin{tabular}{cccc}
\hline Hipótesis & $\mathrm{B}$ & $\begin{array}{c}\text { Valor t } \\
\text { (bootstrap valores }\end{array}$ & $\mathrm{p}$ \\
\hline AC1 - AC2 & 0,604 & 8,573 & 0,000 \\
$\mathrm{AC1}$-> INNMK & 0,168 & 1,483 & 0,138 \\
AC2 - > INNMK & 0,389 & 3,581 & 0,000 \\
ACAP -> INNMK & 0,510 & 7,456 & 0,000 \\
& & \\
$\mathrm{R}^{2}$ Capacidad de ACAP 0,365 & $\mathrm{Q}^{2}$ Capacidad de ACAP 0,203 \\
$\mathrm{R}^{2}$ innovación en marketing 0,260 & $\mathrm{Q}^{2}$ innovación marketing 0,139 & \\
\hline
\end{tabular}

$* * * \mathrm{p}<0,001$ (basado en una distribución t (4999) de Student de una cola)

Fuente: Elaboración propia

Finalmente, la significancia de las relaciones para comprobar las hipótesis del modelo estructural estimadas en los valores t, indica que se aceptan las hipótesis H1, H3 y H4. Sin embargo, se rechaza la hipótesis H2 (Tabla 5).

Tabla 5

Resultados de las hipótesis del modelo estructural

\begin{tabular}{llccc}
\hline & Hipótesis & $\beta$ & Valor t (bootstrap) & CONTRASTE \\
\hline H1 & AC1 $>$ AC2 & 0,604 & $8,573^{* * *}$ & Aceptada \\
H2 & AC1 $>$ INNMK & 0,168 & 1,483 & Rechazada \\
H3 & AC2 $>$ INNMK & 0,389 & $3,851^{* * * *}$ & Aceptada \\
H4 & ACAP $>$ INNMK & 0,510 & $7,456^{* * * *}$ & Aceptada \\
$* * * p<0,001$ (basado en una distribución t (4999) de Student de una cola) & \\
\hline
\end{tabular}

Fuente: Elaboración propia 
Los resultados obtenidos comprueban en la figura 2 que la capacidad de adquisición se conjuga de manera directa con la asimilación del conocimiento, como dimensiones que comprenden la capacidad de absorción potencial, en donde las relaciones de esas variables arrojan un efecto positivo y significativo $(\mathrm{H} 1=8.573)$. Así, la capacidad de absorción potencial genera a su vez, resultados positivos de sobre la innovación en marketing $(\mathrm{H} 4=7.456)$. Y se comprueba, que existe un efecto directo entre la asimilación del conocimiento externo y la innovación $(\mathrm{H}=3.851)$. Finalmente, la única dimensión de la capacidad de absorción potencial que no tiene un impacto significativo es la capacidad de adquisición externa sobre la innovación en marketing $(\mathrm{H} 2=0.168)$.

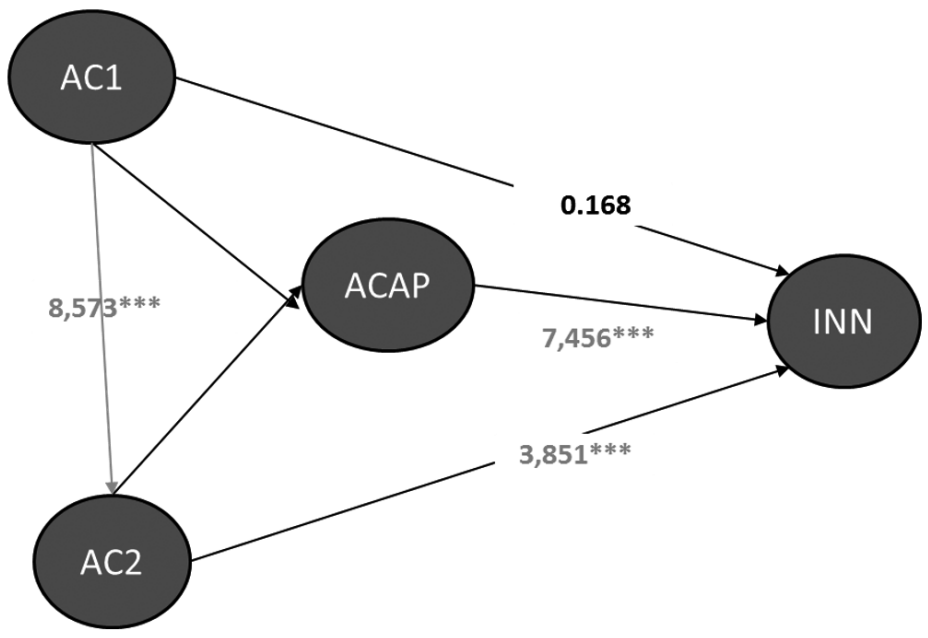

Figura 2. Resultados del análisis PLS para el modelo conceptual. $* * *=\mathrm{p}<0,001$ (basado en una distribución t (4999) de Student de una cola) Fuente: Elaboración propia

\section{Discusiones}

Los hallazgos de esta investigación apoyan en gran medida las hipótesis planteadas. Se evidencio que la interacción entre la capacidad de adquisición y asimilación del conocimiento mejoran la innovación en marketing (Medasa y Barrasa 2019; da Costa et al., 2018) al igual que dichos estudios previos, estos resultados indican que la adquisición de conocimiento 
externo tiene un efecto significativo sobre la asimilación del conocimiento externo (H1) pues, la funcionalidad de adquirir el conocimiento del entorno sobre la capacidad de asimilar el mismo, responde fundamentalmente a la interacción del conocimiento recientemente evaluado, que permite a las empresas incorporar el conocimiento captado del exterior, con base al conocimiento existente en los miembros de la organización (Cohen y Levinthal, 1990), pues tal como plantea Camisón y Forés, (2010) los individuos, así como los equipos trabajo y los grupos de interés interactúan durante los procesos de absorción sobre las fuentes de información externa, que son útiles para las empresas innovar.

Por otra parte, frente a los resultados $\mathrm{H} 2$ se evidencia que la dimensión de la capacidad de absorción potencial del conocimiento que no tiene ningún impacto significativo sobre la innovación en marketing, es la capacidad de adquisición del conocimiento externo, esto podría ser influenciado indirectamente por el diseño e implementación de los planes estratégicos ya establecidos en las empresas turísticas y por aquellas particularidades de este tipo sector, pues gran parte, de la información proviene de fuentes externas (informes gremiales del sector, datos de estatales, investigaciones de universidades, informes del sector privado, etc.) y en el caso de la información de los turista, la connotación transitoria de los mismos podría limitar la identificación de las necesidades reales de los visitantes y aquellas acciones de las empresas frente a los cambios innovadores a concebir. En este sentido, los resultados de este estudio concuerdan con los obtenidos recientemente por Milwood y Zach (2016) quienes determinaron que la relación no significativa entre orientación a la innovación y la adquisición del conocimiento externo, sugiere que la apertura no es un requisito previo para "salir" y encontrar nuevos conocimientos, sino más bien, un precursor para entender cómo los nuevos conocimientos pueden ser útiles para las empresas.

Los resultados encontrados con relación a la relación positiva entre asimilación del conocimiento y la innovación en marketing, se explica por la inmediatez en la prestación del servicio, dada por las competencias previas relacionales de los miembros y la apropiación adecuada del nuevo conocimiento que es percibido como útil en la adopción de las estrategias de las empresas. Tal como indicaron los hallazgos de Milwood y Zach (2016) la capacidad de absorción potencial, se revela en gran medida por la asimilación, por lo que aprender cómo el nuevo conocimiento se ajusta a la organización implica conocer las estructuras, las rutinas organizativas, la cultura, los propósitos organizativos y las estrategias para el correcto aprovechamiento de los nuevos conocimientos. Por lo tanto, los servicios turísticos de nuevo desarrollo deben estar impulsados tanto por la internalización de nuevos conocimientos como por la identificacióny apropiacion de aquellas necesidades que respondan directamente a la generación de valor en las prestaciones de los servicios turísticos. 
Sobre los últimos hallazgos la H4 arrojó una relación significativa de la Capacidad de Absorción Potencial sobre la innovación en marketing, pues al igual que otros estudios la tendencia es que un alto grado de ACAP se relaciona positivamente con el desarrollo de productos más efectivos (Stock et al., 2001) ya que los resultados de la innovación en marketing tiene efectos directos, tales como: incremento de las ventas de productos turísticos, nuevas estrategias de la oferta de servicios, mayor reputación y posicionamiento del portafolio de productos y servicios, así como también, la repetición de la experiencia de los visitantes en el lugar de destino al mediano y largo plazo, generando satisfacción y fidelización de los clientes.

\section{Conclusiones}

El concepto de capacidad de absorción potencial, se puede definir como aquellas habilidades que tienen las empresas de focalizar sus necesidades hacia el entorno, al apropiarse del nuevo conocimiento proveniente de los stakeholders, nutriendo sus capacidades organizativas en acciones asertivas con impacto al mercado.

En primera medida, la evaluación del modelo estructural, permitió la validación de tres de las cuatro hipótesis plantadas. Se demostró que la adquisición del conocimiento externo tiene un efecto significativo en la asimilación del mismo, entendiendo dicha relación como una condición secuencial necesaria para apropiarse de las fuentes de información provenientes de los grupos de interés. Este desencadenamiento en el sector turístico permite reconocer nuevos canales de comercialización y venta del producto o servicio que generaran posteriormente nuevas propuestas de valor en el mercado.

Por otra parte, la capacidad absorber potencialmente el nuevo conocimiento en las empresas turísticas, influye positivamente sobre los resultados de la innovación en marketing, debido a la importancia que ejerce la nueva información proporcionada por turistas, competidores, gremios y entidades gubernamentales, y a la vez, la adopción de la misma en las estrategias de promoción, comercialización y venta de los productos y servicios turísticos.

Se comprobó que la adquisición por sí sola no genera innovación, pues los resultados indicaron que la adquisición del conocimiento no es un consecuente de la innovación en las empresas turísticas, tal como arrojaron los resultados de González-Para Campo y Hurtado Ayala (2014). La no adquisición del conocimiento externo se puede entender porque las estrategias de las empresas están orientadas a satisfacer un segmento especifico del mercado y en mejorar la eficiencia considerando los conocimientos previos, siendo para tal caso, irrelevante el uso de recursos de información externos si no se tiene la apropiación de los mismos. De modo que, si las orientaciones directivas no propician la adquisición del conocimiento externo, mediante actividades de búsqueda, manejo y uso de la información entre los empleados y 
las unidades organizativas, no es probable acceder a nuevos conocimientos que resulten en mejoras o innovación significativas para este tipo de empresas.

Por ello, el generar competitividad de los destinos turísticos, una vez, se establecen interacciones entre las partes interesadas, al adquirir y asimilar el nuevo conocimiento externo permite articular de manera eficiente las estrategias de las empresas con los requerimientos del mercado, generando innovaciones en marketing, delimitando así, las practicas hacia este tipo de resultados que también generan valor a los clientes.

Finalmente, de los resultados obtenidos en relación con las actividades de innovación en marketing, en donde la capacidad de absorción potencial tiene mayor impacto, se pueden extraer algunas implicaciones relevantes. Cabe destacar que las actividades de marketing están vinculadas a estrategias de comercialización, promoción y venta a servicio de la oferta de la industria turística, cuyo personal debe contar con personal idóneo cuyas habilidades y capacidades de comunicación y de servicio al cliente, permitan al adquisición de información pertinente. Por lo tanto, el uso de todas las herramientas digitales y tecnológicas (Software, APP, Redes sociales, Plataformas virtuales, páginas Webs, Videos-influencers, etc.,) amplían la interacción con los stakeholders y promueven el uso de nuevas fuentes de información estableciendo el relacionamiento necesario para identificar, adquirir y asimilar los nuevos recursos en conocimiento útil, que generan nuevas propuestas de valor en el mercado.

Las limitaciones y futuras líneas de investigación del estudio indican la profundización del tema desarrollado desde un enfoque cualitativo, mediante el análisis de estudios de casos que permitan identificar qué tipo de stakeholder generan más oportunidades de innovar y que tipo de innovaciones no tecnológicas promueven el desempeño y la competitividad empresarial de las empresas turísticas.

\section{Referencias}

Akman, G., \& Yilmaz, C. (2008). Innovative capability, innovation strategy and market orientation: an empirical analysis in Turkish software industry. International Journal of Innovation Management, 12(01), 69-111. https:// doi.org/10.1142/S1363919608001923

Ali, M., Kan, K. A. S., \& Sarstedt, M. (2016). Direct and configurational paths of absorptive capacity and organizational innovation to successful organizational performance. Journal of Business Research, 69(11), 53175323. https://doi.org/10.1016/j.jbusres.2016.04.131

Al-Azhar, O., Rose, E. L., \& Chetty, S. (2019). Where to search for process innovations? The mediating role of absorptive capacity and its impact on process innovation. Industrial Marketing Management. 82, $199-212$. https://doi.org/10.1016/j.indmarman.2019.01.014

Amara, N., Landry, R., \& Touré, N. (2008). Managing the protection of innovations in knowledge-intensive business services. Research policy, 37(9), 1530-1547. https://doi.org/10.1016/j.respol.2008.07.00 
Anderson, E. W. (1998). Customer satisfaction and word of mouth. Journal of service research, 1(1), 5-17. https://doi.org/10.1177/109467059800100102

Barbusse, A., y Coenders, G. (2007). Innovation activities, use of appropriation instruments and absorptive capacity: Evidence from Spanish firms. Research Policy, 36(10), 1545-1558. https://doi.org/10.1016/j.respol.2007.04.013

Astro Spila, J., Rocca, L. \& Ibarra, A. (2009). Capacidad de absorción y formas de aprendizaje para la innovación: un modelo conceptual. Projectics / Proyéctica / Projectique, 1(1), 63-76. https://doi.org/10.3917/ proj.001.0063

Awazu, Y. (2006). Managing technology alliances: The case for knowledge management. International Journal of Information Management, 26(6), 484-493. https://doi.org/10.1016/j.ijinfomgt.2006.07.005

Bagozzi R.P. y Yi, Y. (1988). On the evaluation of structural equation models". Journal of the Academy of Marketing Science, 16 (1), pp.74-94. https://doi.org/10.1007/bf02723327

Camison, C. (2005). On how to measure managerial and organizational capabilities: multi-item models for measuring distinctive competences. Management Research: Journal of the Iberoamerican Academy of Management, 3(1), 27-48. https://doi.org/10.1108/15365430580001312

Camisón, C., y Forés, B. (2010). Knowledge absorptive capacity: New insights for its conceptualization and measurement. Journal of Business Research, 63(7), 707-715. https://doi.org/10.1016/j.jbusres.2009.04.022

Cepeda-Carrion, G., Cegarra-Navarro, J. G., y Jimenez-Jimenez, D. (2012). The effect of absorptive capacity on innovativeness: Context and information systems capability as catalysts. British Journal of Management, 23(1), 110-129. https://doi.org/10.1111/j.1467-8551.2010.00725.x

Chin, W. W. (1998): "The partial least squares approach for structural equation modelling”. En George A. Marcoulides (Ed.), Modern Methods for Business Research, Lawrence Erlbaum Associates.

Cohen, W. M., y Levinthal, D. A. (1990). Absorptive capacity: A new perspective on learning and innovation. Administrative science quarterly, 128-152. https://doi.org/10.2307/2393553

Cohendet, P., \& Meyer-Krahmer, F. (2001). The theoretical and policy implications of knowledge codification. Research policy, 30(9), 1563-1591. https://doi.org/10.1016/s0048-7333(01)00168-8 Actions

Corso, M., Martini, A., Pellegrini, L., \& Paolucci, E. (2003). Technological and organizational tools for knowledge management: In search of configurations. Small Business Economics, 21, 397-408.

Cronbach, L. (1951): "Coefficient alpha and the internal structure of tests". Psychometrika, vol. 16, págs. 297334. https://doi.org/10.1007/bf02310555

Cruz-Ros, S., Guerrero-Sánchez, D. L., \& Miquel-Romero, M. J. (2018). Absorptive capacity and its impact on innovation and performance: findings from SEM and fsQCA. Review of Managerial Science, 1-15. https://doi.org/10.1007/s11846-018-0319-7

Eisenhardt, K. M., \& Martin, J. A. (2000). Dynamic capabilities: what are they?. Strategic management journal, 21(10-11), 1105-1121. https://doi.org/10.1002/1097-0266(200010/11)21:10/11<1105::aidsmj133>3.0.co;2-e

Engelman, R.M., Fracasso, E.M., Schnidt, S. \& Zen, A.C. (2017). Intellectual capital, absorptive capacity and product innovation, Management Decision, 55 (3), 474-490. https://doi.org/10.1108/md-05-2016-0315

Escribano, A., Fosfuri, A., y Tribó, J. A. (2009). Managing external knowledge flows: The moderating role of absorptive capacity. Research policy, 38(1), 96-105. https://doi.org/10.1016/j.respol.2008.10.02

Falk, R.F. y Miller, N.B. (1992): A primer for soft modeling. Akron, OH: The University of Akron.

Flatten, T. C., Engelen, A., Zahra, S. A., \& Brettel, M. (2011). A measure of absorptive capacity: Scale development and validation. European Management Journal, 29(2), 98-116. https://doi.org/10.1016/j.emj.2010.11.002 
Forés, B., y Camisón, C. (2016). Does incremental and radical innovation performance depend on different types of knowledge accumulation capabilities and organizational size? Journal of Business Research, 69(2), 831848. https://doi.org/10.1016/j.jbusres.2015.07.006

Fornell,C. y Larcker,D.F.(1981): "Evaluating structural equations models with unobservable variables and measurement error". Journal of Marketing Research, Vol.18, págs.39 50. https://doi.org/10.1177/002224378101800104

Grimpe, C., y Sofka, W. (2009). Search patterns and absorptive capacity: Low-and high-technology sectors in European countries. Research Policy, 38(3), 495-506. https://doi.org/10.1016/j.respol.2008.10.006

González-Campo, C. H., \& Ayala, A. H. (2014). Influencia de la capacidad de absorción sobre la innovación: un análisis empírico en las mipymes colombianas. Estudios Gerenciales, 30(132), 277-286. https://doi.org/10.1016/j.estger.2014.02.015

Guerrero-Sánchez, Diana L. (2017) Impacto de la capacidad de absorción sobre la innovación no tecnológica: análisis del papel moderador del concepto de calidad adoptado por la organización: un estudio en el sector turístico colombiano. Tesis Doctoral. http://roderic.uv.es/handle/10550/61173

Hair, J. F., Ringle, C. M., \& Sarstedt, M. (2011). PLS-SEM: Indeed, a silver bullet. Journal of Marketing theory and Practice, 19(2), 139-152. https://doi.org/10.2753/mtp1069-6679190202

Hair, J., Black, W., Babin, B., Anderson, R. y Tatham, R. (2005): Multivariate Data Analysis, Ed. Prentice Hall, New Jersey.

Henseler, J., Ringle, C.M. y Sinkovics, R.R. (2009): "The use of partial least squares path modeling in international marketing". Advances in International Marketing, 20, 277-320. https://doi.org/10.1108/s1474-7979(2009)0000020014

Hervas-Oliver, J. L., Garrigos, J. A., y Gil-Pechuan, I. (2011). Making sense of innovation by RyD and nonR\&D innovators in low technology contexts: A forgotten lesson for policymakers. Technovation, 31(9), $427-$ 446. https://doi.org/10.1016/j.technovation.2011.06.006

Hervas-Oliver, J. L., y Albors-Garrigos, J. (2009). The role of the firm's internal and relational capabilities in clusters: when distance and embeddedness are not enough to explain innovation. Journal of Economic Geography, 9(2), 263-283. https://doi.org/10.1093/jeg/lbn033

King, B. E., Breen, J., \& Whitelaw, P. A. (2014). Hungry for growth? Small and medium-sized tourism enterprise (SMTE) business ambitions, knowledge acquisition and industry engagement. International Journal of Tourism Research, 16(3), 272-281. https://doi.org/10.1002/jtr.1926

Kostopoulos, K., Papalexandris, A., Papachroni, M., y Ioannou, G. (2011). Absorptive capacity, innovation, and financial performance. Journal of Business Research, 64(12), 1335-1343. https://doi.org/10.1016/j.jbusres.2010.12.005

Lane, P. J., Koka, B. R., y Pathak, S. (2006). The reification of absorptive capacity: A critical review and rejuvenation of the construct. Academy of management review, 31(4), 833-863. https://doi.org/10.5465/amr.2006.22527456

Leal-Rodríguez, A. L., Roldán, J. L., Ariza-Montes J. A., \& Leal-Millán, A. (2014). From potential absorptive capacity to innovation outcomes in project teams: The conditional mediating role of the realized absorptive capacity in a relational learning context. International Journal of Project Management, 32(6), 894-907. https://doi.org/10.1016/j.ijproman.2014.01.005

Leiponen, A., \& Helfat, C. E. (2010). Innovation objectives, knowledge sources, and the benefits of breadth. Strategic management journal, 31(2), 224-236.

Lewin, A. Y., Massini, S., y Peeters, C. (2011). Microfoundations of internal and external absorptive capacity routines. Organization Science, 22(1), 81-98. https://doi.org/10.1287/orsc.1100.0525

Lichtenthaler, U. (2009). Absorptive capacity, environmental turbulence, and the complementarity of organizational learning processes. Academy of management journal, 52(4), 822-846. https://doi.org/10.5465/amj.2009.43670902 
Lynskey, M. J. (2004). Determinants of innovative activity in Japanese technology-based start-up firms. International Small Business Journal, 22(2), 159-196. https://doi.org/10.1177/0266242604041312

Manual Oslo (2005). OECD/European: Communities Guideliness for Collecting and Interpreting Innovation Data, 3d. ed., OECD/EC. Luxembourg. https://doi.org/10.1787/9789264013100-en

Matti Tuominen \& Saara Hyvönen (2004) Organizational Innovation Capability: A Driver for Competitive Superiority in Marketing Channels, The International Review of Retail, Distribution and Consumer Research, 14:3, 277-293. https://doi.org/10.1080/09593960410001678417

Medase, K., \& Barasa, L. (2019). Absorptive capacity, marketing capabilities, and innovation commercialization in Nigeria. European Journal of Innovation Management. https://doi.org/10.1108/ejim-09-2018-0194

Milwood, P., \& Zach, F. (2016). Innovative tourism destinations: Collaboration culture and absorptive capacity. Tourism Travel and Research Association: Advancing Tourism Research Globally. 16. http://scholarworks. umass.edu/tra/2012/Oral/16

Morgan, N. A., Vorhies, D. W., \& Mason, C. H. (2009). Market orientation, marketing capabilities, and firm performance. Strategic Management Journal, 30(8), 909-920. https://doi.org/10.1002/smj.764

Murovec, N., y Prodan, I. (2009). Absorptive capacity, its determinants, and influence on innovation output: Cross-cultural validation of the structural model. Technovation, 29(12), 859-872. https://doi.org/10.1016/j.technovation.2009.05.010

Najafi-Tavani, S., Sharifi, H. and Najafi-Tavani, Z. (2016), "Market orientation, marketing capability, and new product performance: the moderating role of absorptive capacity", Journal of Business Research, Vol. 69 No. 11, pp. 5059-5064. https://doi.org/10.1016/j.jbusres.2016.04.080

Najafi-Tavani, S., Najafi-Tavani, Z., Naudé, P., Oghazi, P., \& Zeynaloo, E. (2018). How collaborative innovation networks affect new product performance: Product innovation capability, process innovation capability, and absorptive capacity. Industrial marketing management, 73, 193-205. https://doi.org/10.1016/j.indmarman.2018.02.009

Nieto, M., y Quevedo, P. (2005). Absorptive capacity, technological opportunity, knowledge spillovers, and innovative effort. Technovation, 25(10), 1141-1157. https://doi.org/10.1016/j.technovation.2004.05.001

Nunnally, J.C. (1978): Psychometric Theory. $2^{\mathrm{a}}$ ed., Mc Graw Hill, Nueva York.

Olaves, G. A., Herrera, L., y Clemenza, C. (2014). Capacidad de absorción: aproximaciones teóricas y empíricas para el sector servicios. Revista Venezolana de Gerencia, 19(67). https://doi.org/10.31876/revista.v19i67.7440

Oliver, C., \& Holzinger, I. (2008). The effectiveness of strategic political management: A dynamic capabilities framework. Academy of Management Review, 33(2), 496-20. https://doi.org/10.5465/amr.2008.31193538

Petraite, M (2010). Knowledge absorption for innovation in new technology based firms: Lithuanian case. In Proc. European Conf. Knowledge Management (ECKM), pp. 767-773.

Rothaermel, F. T., y Alexandre, M. T. (2009). Ambidexterity in technology sourcing: The moderating role of absorptive capacity. Organization science, 20(4), 759-780. https://doi.org/10.1287/orsc.1080.0404

Sarstedt, M., Ringle, C. M., \& Hair, J. F. (2017). Partial least squares structural equation modeling. In Handbook of market research (pp. 1-40). Springer International Publishing. https://doi.org/10.1007/978-3-319-05542-8_15-1

Stock, G.N., Greis, N.P. and Fischer, W.A. (2001). Absorptive capacity and new product development. Journal of High Technology Management Research, 12, 77-91 https://doi.org/10.1016/s1047-8310(00)00040-7

Tejada, P., Moreno, P., \& Rodríguez, M. J. (2013). Contribución de las pymes turísticas al desarrollo. rEviSta dE Economía mundial, (35), 103-119. Disponible en: https://www.redalyc.org/pdf/866/86629567005.pdf 
Tidd, J., \& Bessant, J. (2018). Innovation management challenges: From fads to fundamentals. International Journal of Innovation Management, 22(05), 1840007. https://doi.org/10.1142/S1363919618400078

Thomas, R., y Wood, E. (2015). The absorptive capacity of tourism organisations. Annals of Tourism Research, 54, 84-99. https://doi.org/10.1016/j.annals.2015.06.004

Vargo, SL, H Wieland and MA Akaka (2015). Innovation through institutionalization: A service ecosystems perspective. Industrial Marketing Management, 44, 63-72 https://doi.org/10.1016/j.indmarman.2014.10.008

Tzokas, N., Kim, Y.A., Akbar, H. and Al-Dajani, H. (2015), "Absorptive capacity and performance: the role of customer relationship and technological capabilities in high-tech SMEs", Industrial Marketing Management, Vol. 47, pp. 134-142 https://doi.org/10.1016/j.indmarman.2015.02.033

Vázquez, S. Y. S., Fernández, F. G., \& Félix, M. Z. (2017). Impacto de la capacidad de absorción del conocimiento en la innovación. El caso del sector petroquímico en Reynosa, México. Innovar: Revista de ciencias administrativas y sociales, 27(66), 11-27 https://doi.org/10.15446/innovar.v27n66.66708

Volberda, H. W., Foss, N. J., \& Lyles, M. A. (2010). Perspective-Absorbing the concept of absorptive capacity: How to realize its potential in the organization field. Organization science, 21(4), 931-951. https://doi.org/10.1287/orsc.1090.0503

Williams, A. M., \& Shaw, G. (2011). Internationalization and innovation in tourism. Annals of Tourism Research, 38(1), 27-51. https://doi.org/10.1016/j.annals.2010.09.006

Werts, C.E., Linn, R.L. y Jöreskog, K.G. (1974): "Interclass reliability estimates: testing structural assumptions". Educational and Psychological Measurement, 34, 25-33. https://doi.org/10.1177/001316447403400104

West, J., \& Bogers, M. (2017). Open innovation: current status and research opportunities. Innovation, 19(1), 4350. https://doi.org/10.1080/14479338.2016.1258995

Williams, A.M. and Shaw, G. (2011). Internationalization and innovation in tourism. Annals of Tourism Research, 38(1), 27口51. https://doi.org/10.1016/j.annals.2010.09.006

Xie, X., Zou, H., \& Qi, G. (2018). Knowledge absorptive capacity and innovation performance in high-tech companies: A multi-mediating analysis. Journal of Business Research, 88, 289-297. https://doi.org/10.1016/j.jbusres.2018.01.019

Zach, F. (2012). Partners and Innovation in American Destination Marketing Organizations. Journal of Travel Research, 51(4), 412-425. https://doi.org/10.1177/0047287511426340

Zahra, S. A., y George, G. (2002). Absorptive capacity: A review, reconceptualization, and extension. Academy of management review, 27(2), 185-203. https://doi.org/10.5465/amr.2002.6587995

Zobel, A. K. (2017). Benefiting from open innovation: A multidimensional model of absorptive capacity. Journal of Product Innovation Management, 34(3), 269-288. https://doi.org/10.1111/jpim.1236 


\section{Anexo}

Desde la Universidad de valencia (España) en colaboración con la Fundación Universitaria Lumen Gentium-Unicatólica, y con el apoyo de las agremiaciones de turismo como COTEL$\mathrm{CO}$, estamos realizando un estudio sobre el impacto de la capacidad de absorción potencial del conocimiento sobre la innovación en marketing de las empresas del sector turístico colombiano. Amablemente los invitamos a participar en tan importante proceso con impacto a nivel nacional y para el despliegue de posteriores estudios en paralelo con España. Para ello, les solicitamos al propietario de las empresas asociadas, el gerente o la persona encargada de coordinar la gestión de marketing en concedernos 10 minutos de su tiempo para contestar las preguntas vía online.

A continuación, se le realizan una serie de preguntas para las que no existen respuestas buenas o malas. La respuesta adecuada es aquella que mejor describe el estado actual de su organización, de antemano le agradecemos su tiempo y valiosa colaboración.

\section{DATOS DE LA EMPRESA: V1-V5}

\begin{tabular}{lll|l|l|}
\cline { 3 - 4 } V1 ¿Cuántos & años de & Menos de 5 años & 1 \\
\cline { 4 - 4 } funcionamiento & lleva su & Entre 5 -10 años & 2 \\
\cline { 4 - 4 } & & & $\begin{array}{l}\text { Entre 10- 20 años } \\
\text { Más de 20 años }\end{array}$ & 3 \\
\cline { 4 - 4 } & & &
\end{tabular}

V3 Tipo de empresa?

\begin{tabular}{|l|l|l|}
\hline 1 & Hoteles & \\
\hline 2 & Restaurantes & \\
\hline 3 & $\begin{array}{l}\text { Agencia de } \\
\text { viajes }\end{array}$ & \\
\hline 5 & Otra. ¿Cuál? & \\
\hline
\end{tabular}

V2 ¿Número total de empleados fijos a tiempo completo?

\begin{tabular}{|l|l|l|}
\hline 1 & $1-10$ & \\
\hline 2 & $11-50$ & \\
\hline 3 & $51-200$ & \\
\hline 4 & Más de 200 & \\
\hline
\end{tabular}

V4 Asociación a la que pertenece?
\begin{tabular}{|l|l|l|}
\hline 1 & Acodres & \\
\hline 2 & Anato & \\
\hline 3 & Asotelca & \\
\hline 4 & Cotelco & \\
\hline 5 & Otra. ¿Cuál? & \\
\hline
\end{tabular}

V5. Ciudad
Cali
Bogotá
Medellín
Barranquilla
Cúcuta
Otras 


\section{CAPACIDAD DE ABSORCIÓN POTENCIAL (CA V6-V12)}

Por favor especifique en qué medida las siguientes afirmaciones permiten que su empresa utilice recursos externos para la adquisición de conocimientos al obtener información de redes personales, clientes, proveedores, competidores, consultores y demás grupos de interés, siendo:

\section{Totalmente en desacuerdo, 2. En desacuerdo, 3. Indiferente, 4 De acuerdo,} y 5 Totalmente de acuerdo.

\begin{tabular}{|c|c|c|c|c|c|c|}
\hline & & 1 & 2 & 3 & 4 & 5 \\
\hline V6 & $\begin{array}{l}\text { La búsqueda de información relevante sobre la industria es una } \\
\text { actividad diaria en la empresa }\end{array}$ & & & & & \\
\hline V7 & $\begin{array}{l}\text { La dirección de la empresa motiva a los empleados a utilizar fuentes } \\
\text { de información dentro de la industria }\end{array}$ & & & & & \\
\hline V8 & $\begin{array}{l}\text { La dirección de la empresa espera que los empleados manejen infor- } \\
\text { mación más allá de la industria. }\end{array}$ & & & & & \\
\hline
\end{tabular}

Indique en qué medida las siguientes afirmaciones se ajustan a la estructura de comunicación para la asimilación de conocimiento en su empresa:

\begin{tabular}{|c|c|c|c|c|c|c|}
\hline & & 1 & 2 & 3 & 4 & 5 \\
\hline V9 & En la empresa las ideas son comunicadas entre los departamentos. & & & & & \\
\hline V10 & $\begin{array}{l}\text { La dirección destaca por el apoyo entre departamentos para resolver pro- } \\
\text { blemas. }\end{array}$ & & & & & \\
\hline V11 & $\begin{array}{l}\text { En la empresa existe un flujo de información rápido, por ejemplo, si una } \\
\text { unidad de negocio obtiene información importante esta se comunica a todas } \\
\text { las demás unidades o departamentos de la empresa }\end{array}$ & & & & & \\
\hline V12 & $\begin{array}{l}\text { La dirección exige reuniones periódicas interdepartamentales para inter- } \\
\text { cambiar novedades, problemas y logros. }\end{array}$ & & & & & \\
\hline
\end{tabular}




\title{
INNOVACIÓN (INNMK V13-V19)
}

\author{
Por favor, indique el grado en que se han introducido los siguientes elementos en canales de \\ ventas o distribución nuevos.
}

Totalmente en desacuerdo, 2. En desacuerdo, 3. Indiferente, 4 De acuerdo, y 5 Totalmente de acuerdo.

\begin{tabular}{|c|c|c|c|c|c|c|}
\hline & & 1 & 2 & 3 & 4 & 5 \\
\hline V13 & Modificación significativa de los métodos de comercialización actuales & & & & & \\
\hline V14 & Reducción del periodo de respuesta a un cliente o proveedor & & & & & \\
\hline V15 & Cambios significativos en el diseño del servicio & & & & & \\
\hline V16 & Cambios significativos en la tarificación de los productos/servicios & & & & & \\
\hline V17 & $\begin{array}{l}\text { Introducción de nuevos canales y formatos para promocionar produc- } \\
\text { tos/servicios }\end{array}$ & & & & & \\
\hline V18 & $\begin{array}{l}\text { Desarrollo y lanzamiento de nuevas marcas, con el fin de renovar ima- } \\
\text { gen o posicionarse en nuevos mercados }\end{array}$ & & & & & \\
\hline V19 & Renovación de las marcas existentes & & & & & \\
\hline
\end{tabular}

V20. ¿Dispone su empresa de un departamento específico en Innovación?

1- $\mathbf{S I}$

2- NO 